\title{
Hybrid III-V Silicon Photonic Crystal Cavity Emitting at Telecom Wavelengths
}

Svenja Mauthe, Preksha Tiwari, Markus Scherrer, Daniele Caimi, Marilyne Sousa, Heinz Schmid, Kirsten E. Moselund, and Noelia Vico Triviño*

IBM Research Europe, Säumerstr. 4, 8803 Rüschlikon

\begin{abstract}
Photonic crystal (PhC) cavities are promising candidates for Si photonics integrated circuits due to their ultrahigh quality $(Q)$-factors and small mode volumes. Here, we demonstrate a novel concept of a one-dimensional hybrid III-V/Si PhC cavity which exploits a combination of standard siliconon-insulator technology and active III-V materials. Using template-assisted selective epitaxy, the central part of a $\mathrm{Si} \mathbf{P h C}$ lattice is locally replaced with III-V gain material. The III-V material is placed to overlap with the maximum of the cavity mode field profile, while keeping the major part of the $\mathrm{PhC}$ in $\mathrm{Si}$. The selective epitaxy process enables growth parallel to the substrate and hence, inplane integration with $\mathrm{Si}$, and in-situ in-plane homo- and heterojunctions. The fabricated hybrid III$\mathrm{V} / \mathrm{Si}$ PhCs show emission over the entire telecommunication band from $1.2 \mu \mathrm{m}$ to $1.6 \mu \mathrm{m}$ at room temperature validating the device concept and its potential towards fully integrated light sources on silicon.
\end{abstract}

The field of photonics is very promising enabling high-speed and low power optical interconnects as well as added functionalities. Especially Si photonic integrated circuits (PICs) are of great interest, allowing the use of existing low-cost and mature $\mathrm{Si}$ technology and enabling an ideal combination of electronics for computation and optics for highspeed data transmission $[1,2]$. While efficient onchip passive and active photonic devices have been demonstrated using $\mathrm{Si}$, its indirect band gap prevents efficient light emission. To create a full optical PIC, other materials have to be integrated that enable efficient light sources like lasers and light emitting diodes (LEDs). Driven by this demand, extensive research has been carried out to integrate III-Vs on $\mathrm{Si}$ towards integrated light sources [3-7]. III-V materials offer a direct and tunable band gap in the telecommunication band, and hence, are suitable for the use with Si passives. The challenge however is the monolithic integration on $\mathrm{Si}$ due to a mismatch of the crystal lattice, polarity, and thermal expansion coefficient.

Photonic crystal (PhC) cavities have emerged as ideal candidates for small footprint integrated light sources $[8,9]$. They offer ultrahigh quality $(Q)$ factors combined with small mode volumes $(V)$, resulting in an enhancement of the light-matter 
interaction at the nanoscale. Extremely high $Q / V$ ratios are featured in the so-called one-dimensional (1D) nanobeam cavities $[10,11]$. Typical $Q \mathrm{~s}$ on the order of $10^{6}$ and mode volumes approaching the diffraction limit $(\lambda / 2 n)^{3}$ are widely reported $[3,11$, 12] even with low refractive index materials such as SiN [13]. This is a key advancement since it overcomes the need of freestanding structures $(\mathrm{PhC}$ slabs) and enables $\mathrm{Si}$ embedded in $\mathrm{SiO}_{2}$ structures. In contrast with $\mathrm{PhC}$ slabs, structures with $\mathrm{Si}$ embedded in $\mathrm{SiO}_{2}$ are mechanically more robust, can be coupled in-plane with silicon-on-insulator (SOI) waveguides and passives, and offer a more efficient thermal management [14]. PhCs also offer great design flexibility that can be exploited in many applications. They have demonstrated unique properties for the realization of lasers with highspeed modulation rates [15-17] and low thresholds [3, 18-20]. Indeed, for ideal PhC cavities thresholdless lasing has been theoretically predicted [21, 22]. The performance of $\mathrm{PhC}$ lasers is typically limited by poor carrier confinement and inefficient heat dissipation. The use of a small active gain medium located in the $\mathrm{PhC}$ cavity has proven to be very effective to address such challenges [17, 23, 24]. Different approaches have been proposed such as selective regrowth techniques to create an ultracompact buried InGaAsP heterostructure at the center of an $\mathrm{InP} \mathrm{PhC}$ [17] and pick-and-place of an InAsP/InP nanowire on a Si PhC [23, 24]. While impressively demonstrating the potential of local embedding of a gain material within a $\mathrm{PhC}$, fabrication issues, like the precise placement of such gain material, remain very challenging.

This work demonstrates a novel concept of a 1D III$\mathrm{V} /$ Si hybrid photonic crystal cavity where the gain emitting medium (III-V) is embedded locally within a silicon photonic crystal on standard SOI wafers. The key idea of the proposed concept is to selectively replace parts of the $\mathrm{Si} \mathrm{PhC}$ with active III-V materials using template-assisted selective epitaxy (TASE) [25-29]. In TASE, the III-V material is epitaxially grown from a small $\mathrm{Si}$ seed into a prefabricated, hollow $\mathrm{SiO}_{2}$ cavity. The selective replacement of $\mathrm{Si}$ structures by III-Vs naturally results in a self-aligned process with high dimensional accuracy and enables direct in-plane coupling to $\mathrm{Si}$ waveguides. Such a growth approach enables the integration of different III-V materials [30] as well as the creation of as-grown $p-i-n$ structures parallel to the substrate [29], making exsitu diffusion or implantation doping redundant.

Fig. 1 shows the concept of the 1D hybrid III-V / Si $\mathrm{PhC}$ cavity. The cavity consists of a periodic array of high refractive index rods ( $\mathrm{Si}$ or III-Vs) embedded in a low refractive index dielectric $\left(\mathrm{SiO}_{2}\right)$ as depicted in Fig. 1 (b). In this work, we make use of the Sibased $\mathrm{PhC}$ design reported in [12] due to its favorable geometry to study and prove our hybrid concept. In principle, other designs can also be implemented with the same approach. This Si 1D PhC cavity features an ultra-high $Q$-factor $\left(>10^{6}\right)$ thanks to a quadratically modulated length tapering of the individual rods and can be straightforwardly coupled to waveguides. In the hybrid structure the gain material is located to overlap with the maximum of the electric field profile of the cavity mode (Fig. 1 (c)). Simultaneously, cavity losses are reduced, since the Si mirrors do not absorb in the same wavelength range as the emission. To facilitate a match to the original all-Si design [12], we extend the layout by varying the width of the III-V rods to compensate for slight differences in the refractive indices $n_{\mathrm{Si}}$ and $n_{\mathrm{III}-\mathrm{V}}$ of $\mathrm{Si}$ and III-Vs, respectively. Thus, by adjusting the width of the III-V rods, the hybrid $\mathrm{PhC}$ can exhibit a similar $Q$-factor and resonance wavelength as compared to the original all-Si design. The total number of rods (Si and III$\mathrm{V})$ is kept constant $(\mathrm{N}=41)$ while the number of III$\mathrm{V}$ rods is either 5 or 9 to overlap with the maximum or the entire mode profile, respectively (see Fig. 1(c)). Moreover, a sweep of the PhC lattice constant $a$ (distance between two adjacent rods) from $325 \mathrm{~nm}$ to $400 \mathrm{~nm}$ is included. The III-V rods consist of a 
(a) Hybrid III-V / Si photonic crystal cavity

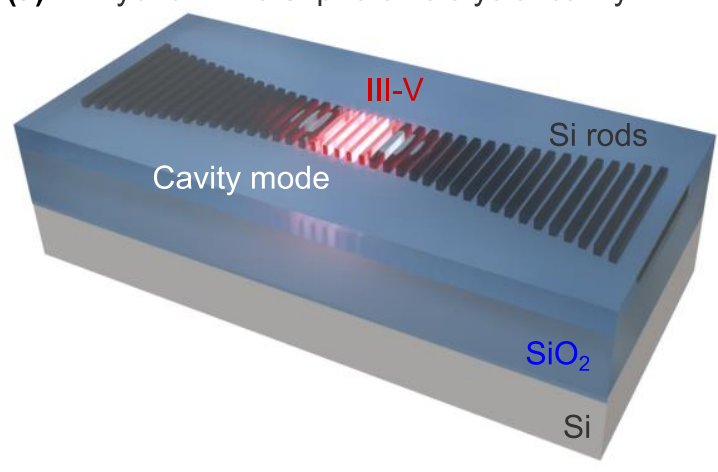

(b)

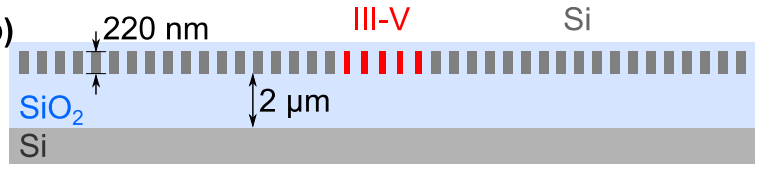

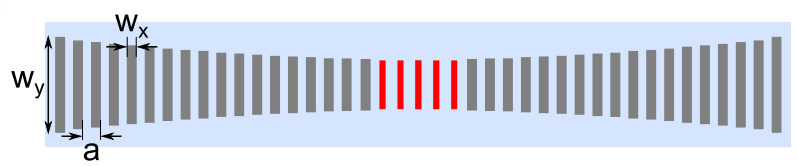

(c)

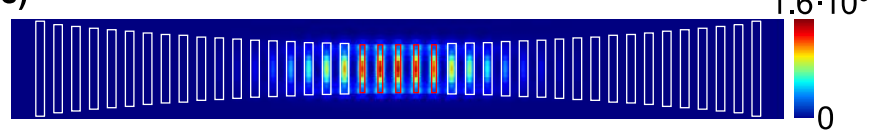

Fig. 1 (a) 3D view of the hybrid III-V/ Si PhC cavity integrated on SOI. (b) Cross-section (top) and top view (bottom) schematic of the hybrid cavity. The rods are length modulated according to $w_{y}(i)=w_{y}(0)+i^{2}\left(w_{y}\left(i_{\max }\right)-w_{y}(0)\right) / i_{\max }{ }^{2}$ where $w_{y}(i)$ is the length of the $i^{\text {th }}$ rod. The width $w_{x}$ is kept constant. Both, $w_{y}$ and $w_{x}$ are proportional to the lattice constant a. (c) IEy/ $\left.\right|^{2}$ profile of the first cavity mode calculated via $3 D$ FDTD.

heterostructure including $\mathrm{InP} / \mathrm{InGaAs} / \mathrm{InP}$ designed length of the middle rods in the PhC cavity. segments which are additionally in-situ doped The remaining Si acts as a nucleation seed during IIIduring growth to form a $p-i-n$ diode to resemble as $\mathrm{V}$ growth in step 5 . We note that the presence of the close as possible an electrically actuated device back-etched Si rods, which serve as growth seeds, configuration. This allows tuning of the emission and the oxide window to reach the rods slightly wavelengths ranging from $1.2 \mu \mathrm{m}$ to $1.6 \mu \mathrm{m}$, i.e., break the design symmetry of the PhC. This, covering the entire telecom window.

Fig. 2 depicts the individual processing steps to create the hybrid III-V/Si PhC cavity. The fabrication starts with a commercial SOI wafer (SOITEC) with a $2 \mu \mathrm{m}$ thick buried oxide (BOX) for sufficient optical isolation and a $220 \mathrm{~nm}$ thick top $\mathrm{Si}(100)$ layer. In a first step, the top Si layer is patterned by means of e-beam lithography and $\mathrm{HBr}$ dry etching. Next, $\mathrm{SiO}_{2}$ is deposited, $100 \mathrm{~nm}$ using atomic layer deposition (ALD) and $200 \mathrm{~nm}$ by plasma-enhanced chemical vapor deposition (PECVD). The deposited oxide layer is planarized using chemical mechanical polishing (CMP) and reduced to a thickness of $150 \mathrm{~nm}$. Next, windows are etched into the oxide layer exposing one end of the targeted Si rods in the middle of the cavity. The exposed $\mathrm{Si}$ is partly etched using tetramethylammonium hydroxide (TMAH). The etch rate is precise on a nm-scale and is adjusted via the temperature and concentration of the TMAH solution. Step 4 in Fig. 2 schematically depicts the partially etched Si rods and the remaining empty $\mathrm{SiO}_{2}$ template. The etch is timed to match the however, is not a limiting factor of the concept and can be optimized by e.g. performing a second lithography. Metal-organic chemical vapor deposition (MOCVD) is used to grow III-V materials selectively from the $\mathrm{Si}$ seeds into the empty $\mathrm{SiO}_{2}$ templates (Fig 2, step 5). Shortly before the growth, a $20 \mathrm{~s}$ dilute hydrofluoric acid (DHF) etch is performed to remove native oxide on the $\mathrm{Si}$ seeds, during which the $\mathrm{SiO}_{2}$ cavity is enlarged by $30 \mathrm{~nm}$. The $n$-InP / $i$-InGaAs / $p$-InP / $p$-InGaAs heterojunction are grown at $550^{\circ} \mathrm{C}$ using TMIn, TBP, TBAs, TMGa with DEZn and TESn as $p$ - and $n$-type dopants. The additional outer $p$-InGaAs
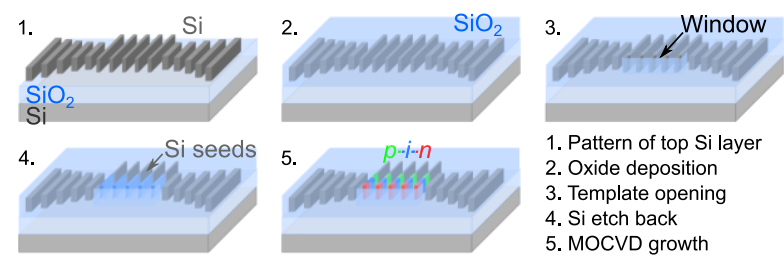

Fig. 2 Fabrication steps during TASE process. Starting from a standard SOI wafer, the top Si is patterned according to the PhC design (1.). 2. Oxide is deposited, and the sample is planarized. 3. Template opening exposing the underlying Si for the center rods. 4. Partial etch of the Si rods in the center. 5. MOCVD growth of $n-i-p \operatorname{InP}$ / InGaAs heterostructure. 
segment was added to mimic a future contact layer to the $p$-InP segment. The fabrication of electrical contacts is out of scope of this initial demonstration and might require an optimization of the hybrid $\mathrm{PhC}$ cavity.

Fig. 3 (a) and (b) depict scanning electron microscopy (SEM) overview images of two hybrid $\mathrm{PhC}$ cavities with 5 and 9 III-V rods, respectively. A substantial variation in the length of the grown III-V rods is observed, which is an indication of nonuniform nucleation and likely due to residues on the Si surface. This is not intrinsic to the growth method. Fig. 3 (c) shows an SEM top-view image of a single III-V rod. Fig. 3 (d) depicts a cross-section along the center of the $\mathrm{PhC}$ cavity which demonstrates the hybrid integration and illustrates the alignment of the III-V rods with the Si rods. To further investigate the material quality of the grown devices, scanning transmission electron microscopy (STEM) analysis is performed as shown in Fig. 3 (e) and (f). The highresolution images confirm a single crystalline material and epitaxy relationship with the Si layer.

The hybrid $\mathrm{PhC}$ cavities are characterized by microphotoluminescence ( $\mu$-PL) spectroscopy. The cavities are illuminated with an $850 \mathrm{~nm}$ ps-pulsed supercontinuum laser (repetition rate: $78 \mathrm{MHz}$, spot size: $\sim 1 \mu \mathrm{m}$ ) to optically excite the grown III-V
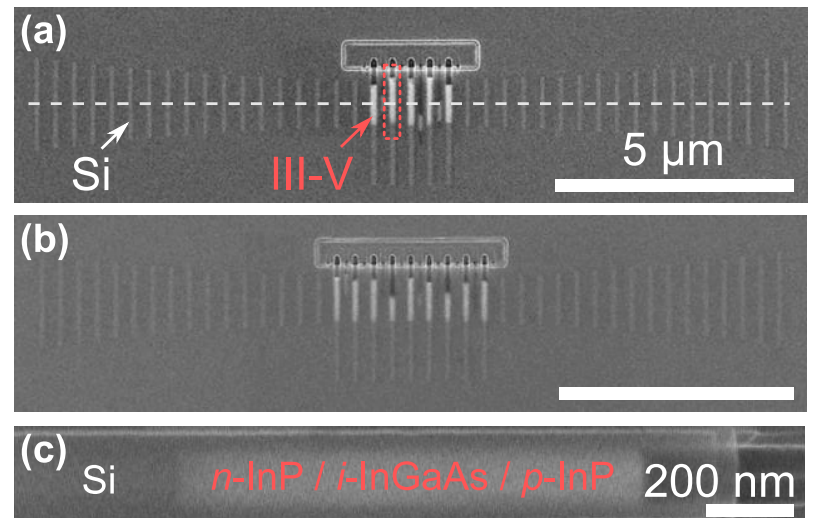

material. Both, the excitation and response of the sample, are focused and collected from the top using a $\times 100$ objective (NA 0.6 ). The optical response is characterized using a spectrometer with a liquid nitrogen cooled InGaAs linear array photodetector. The PhC structure exhibits a spontaneous emission background starting from $1.2 \mu \mathrm{m}$ to $1.6 \mu \mathrm{m}$ along with the different cavity modes. The gain emitting material allowed us to observe cavity modes over the entire telecom window, by changing the lattice constant $a$ of the PhC. Fig. 4 (a) shows the PL spectra of hybrid PhC cavities with 5 III-V rods and varying a. Three cavity modes are visible. Fig. 4 (b) depicts the measured center wavelength versus $a$ of the optical modes for hybrid PhC cavities with 5 and 9 rods in the center of the hybrid cavity. The resonances shift to longer wavelengths with increasing $a$ demonstrating full tunability over the telecom range and hence, enabling emission in the relevant $\mathrm{O}$ - and $\mathrm{C}$-bands. It is worth mentioning that despite the non-uniform length of the III-V rods, we observe similar behavior for all the measured cavities, with similar experimental $Q$-factors on the order of $10^{3}$. This is illustrated in Fig. 4 (b) for 17 different structures. No significant differences were observed with regard to the number of III-V rods (5 or 9). We believe that the observed robustness and high yield in such non-perfect $\mathrm{PhC}$ cavities relies on
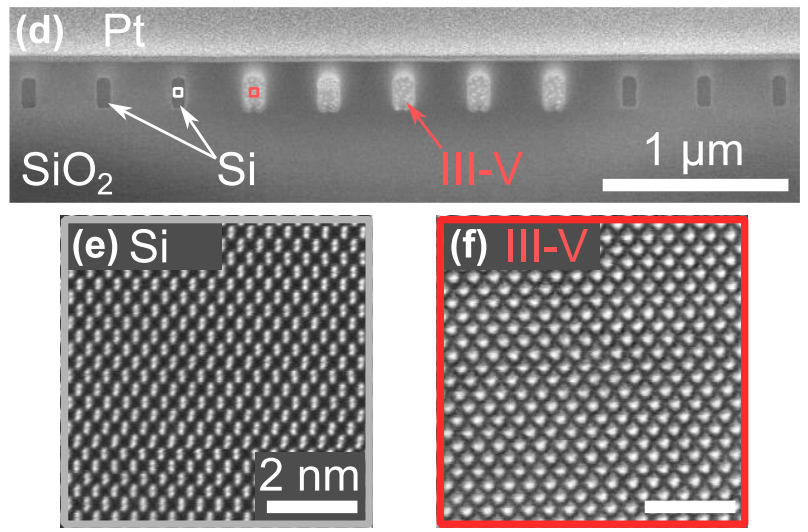

Fig. 3 (a) SEM images of hybrid PhC cavity with 5 (a) and 9 (b) III-V rods surrounded by Si mirrors. (c) SEM image of a single III-V rod marked in (a). (d) STEM cross-section of a hybrid PhC cavity with 5 rods along the $x$-axis of the hybrid PhC cavity (dashed line in (a)). The III-V rods are aligned in between the Si rods of the hybrid PhC cavity. (e) \& (f) High resolution STEM images of the Si (e) and grown III-V (f) rods at marked positions in (d). Although only the first III-V rod is selected here, it is representative of the 4 remaining ones. 
(a)

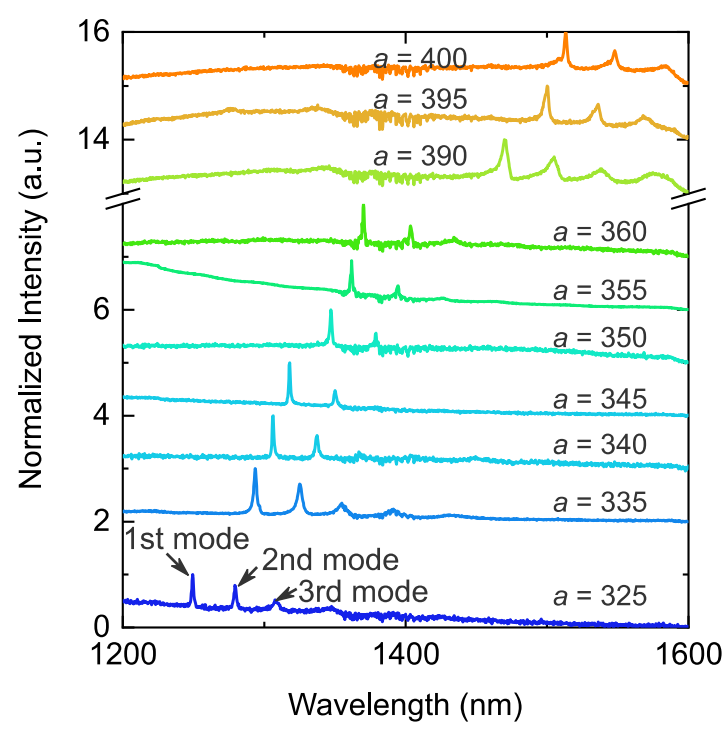

(b)

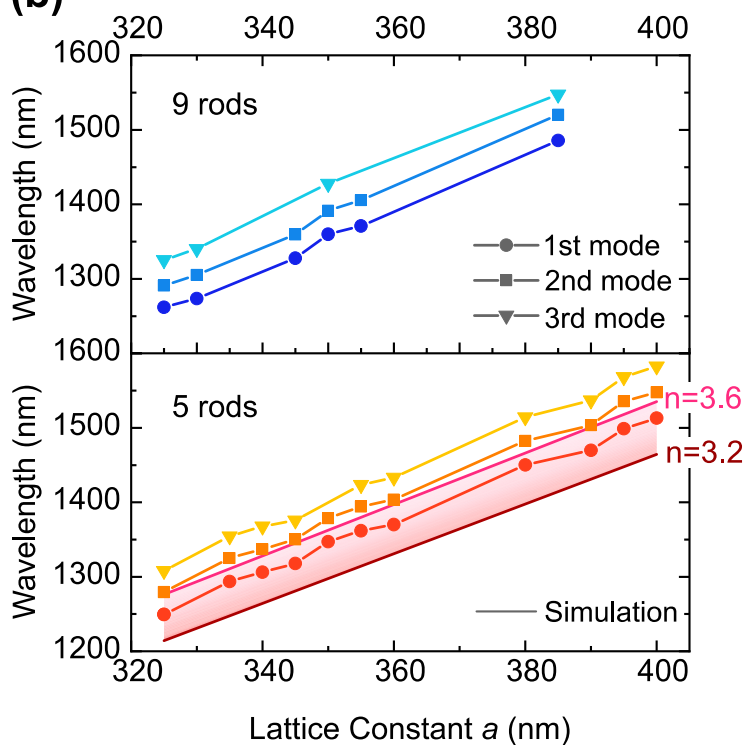

Fig. 4 (a) PL spectrum of hybrid PhC cavities with 5 rods for increasing lattice constant a $\left(w_{x}=0.33 a, w_{y}=5 a\right)$. (b) Measured peak wavelength of the three resonant modes versus lattice constant of the hybrid PhC plotted for two different number of III-V rods: 5 and 9 in the hybrid cavity $\left(w_{x}=0.33 a, w_{y}=5 a\right)$. One can observe cavity resonances spanning the entire telecom spectral range. The shadow area encloses the peak wavelengths of the first cavity mode obtained by $3 D$-FDTD simulations using refractive index values of $n_{I I I-V}$ between 3.2 and 3.6 .

the local integration of III-Vs within the central part of an otherwise ideal Si lattice of our hybrid $\mathrm{PhC}$.

We perform 3D FDTD simulations using Lumerical FDTD Solutions. Fig. 4(b) depicts the simulated wavelength shift over lattice constant of the first cavity mode. The lower and upper limits of the shadow area correspond to the resonance wavelengths for constant values of the III-V refractive index of 3.2 and 3.6, respectively [31]. Owing to the complexity of our III-V rods combining InP and InGaAs, estimating an exact refractive index dispersion curve or value is not trivial. The simulated trend (shadow area) agrees well with the experimental data (red circles).

Fig. 5 (a) depicts the spectra of a hybrid PhC cavity under increasing optical excitation. With increasing excitation fluence, the light intensity of the resonances as well as the spontaneous emission background, increase. Fig. 5 (b) shows the integrated optical power (light in-light out (LL) curve) of the cavity mode at $1368.5 \mathrm{~nm}$ for increasing pump powers. Fig. 5 (c) depicts the full width at half maximum (FWHM) of the resonant peak as well as the LL curve in log-log scale. The FWHM decreases from $\sim 5.6 \mathrm{~nm}$ at low excitation powers to $\sim 1.8 \mathrm{~nm}$. The slight increment in the FWHM at very high excitation fluence is observed due to plasma dispersion effect which result in a blueshift of the peak $[32,33]$.

We believe that the observed linewidth narrowing and measured LL curve show evidence of lasing in our structures at room temperature. However, to unambiguously demonstrate lasing in such structures, further studies of carrier lifetime and photon statistics (correlation measurements) are required. This goes beyond the scope of this proofof-concept paper.

In summary, we demonstrated a novel method to create a hybrid III-V / Si light source on SOI with tunable operation over the entire telecom band at room temperature. The hybrid $\mathrm{PhC}$ crystal concept along with the presented fabrication technique benefits from the use of mature $\mathrm{Si}$ processing technology and existing Si PhC designs. The local 

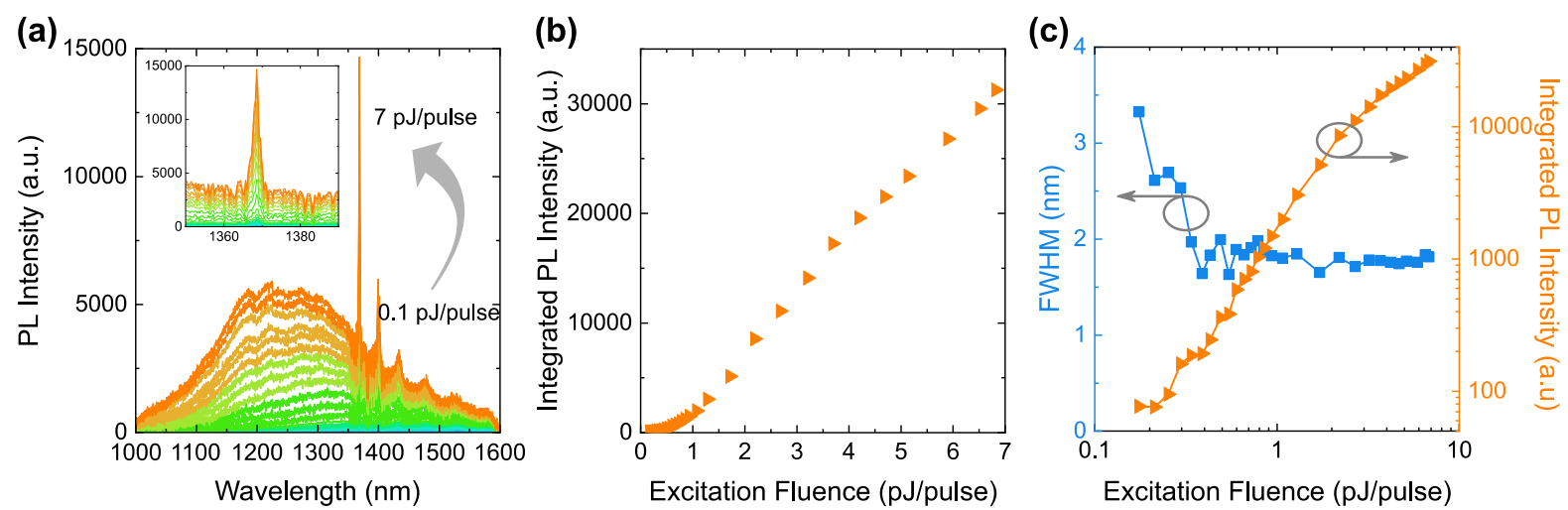

Fig. 5 (a) Measured PL intensity of a hybrid PhC for increasing excitation fluences $\left(a=375 \mathrm{~nm}, 5\right.$ rods, $\left.w_{x}=0.3 a, w_{y}=5 a\right)$. (b) Integrated PL intensity versus excitation fluence of the resonant peak at $1368 \mathrm{~nm}$ in (a). (c) FWHM and integrated PL intensity from (b) versus excitation fluence in double log scale.

embedding of the III-Vs on Si takes place seamlessly passives. The III-V gain material was implemented in the very last step of the fabrication process. Thus, as lateral heterostructures with an in-situ as-grown one can foresee fabrication of the full $\mathrm{Si} \mathrm{PhC}$ in a $p$-i-n doping profile [34] to illustrate a path towards silicon foundry together with Si waveguides and electrically driven, integrated light source on silicon.

\section{Corresponding Author}

*nvi@zurich.ibm.com

\section{Author Contributions}

S.M., P.T., M.S., H.S., K.M., and N.V.T. created the device concept. N.V.T. performed FDTD simulations. S.M. and P.T. fabricated the sample with support of D.C. for the CMP. S.M. performed the optical characterization on the devices. S.M., N.V.T., and P.T. analyzed the data. M.Sousa performed the FIB lamella and STEM characterization. K.M., and N.V.T. lead and managed the project. All authors discussed the results. The manuscript was written by S.M. and N.V.T., with contributions of all authors, and all authors have given approval to the final version of the manuscript.

\section{Funding Sources}

This work received financial support from H2020 ERC project PLASMIC, Grant \#678567 and Swiss National Science Foundation Spark project SPILA, Grant \#CRSK-2_190806.

\section{ACKNOWLEDGMENT}

The authors gratefully acknowledge the BRNC staff for technical support.

\section{ABBREVIATIONS}

PhC photonic crystal, TASE template-assisted selective epitaxy, PIC photonic integrated circuits, LED light emitting diode, SOI silicon-on insulator, BOX buried oxide, ALD atomic layer deposition, PECVD plasma- 
enhanced chemical vapor deposition, CMP chemical mechanical polishing, TMAH tetramethylammonium hydroxide, MOCVD metal-organic chemical vapor deposition, DHF dilute hydrofluoric acid, TMIn Trimethylindium, TBP Tertiarybutylphosphine, TBAs Tertiarybutylarsine, TMGa Trimethylgallium, DEZn Diethylzinc, TESn Tetraethyltin, SEM scanning electron microscopy, EDX energy-dispersive X-ray spectroscopy, STEM scanning transmission electron microscopy, FFT Fast Fourier transform, PL

photoluminescence, NA numerical aperture, LL light in-light out, FWHM full width at half maximum, FDTD finite-difference-time-domain.

\section{REFERENCES}

[1] C. Sun et al., "Single-chip microprocessor that communicates directly using light," Nature, vol. 528, no. 7583, pp. 534-538, Dec. 2015, doi: 10.1038/nature16454.

[2] A. H. Atabaki et al., "Integrating photonics with silicon nanoelectronics for the next generation of systems on a chip," Nature, vol. 556, no. 7701, pp. 349-354, Apr. 2018, doi: 10.1038/s41586-018-0028-Z.

[3] G. Crosnier et al., "Hybrid indium phosphide-on-silicon nanolaser diode," Nat. Photonics, vol. 11, no. 5, pp. 297-300, 2017, doi: 10.1038/nphoton.2017.56.

[4] B. Shi et al., "1.55 $\mu \mathrm{m}$ room-temperature lasing from subwavelength quantum-dot microdisks directly grown on (001) Si," Appl. Phys. Lett., vol. 110, no. 12, p. 121109, 2017, doi: $10.1063 / 1.4979120$.

[5] B. Kunert et al., "III/V nano ridge structures for optical applications on patterned $300 \mathrm{~mm}$ silicon substrate," Appl. Phys. Lett., vol. 109, no. 9, 2016, doi: 10.1063/1.4961936.

[6] G. Morthier, T. Spuesens, P. Mechet, G. Roelkens, and D. Van Thourhout, "InP Microdisk Lasers Integrated on Si for Optical Interconnects," IEEE J. Sel. Top. Quantum Electron., vol. 21, no. 6, pp. 359-368, Nov. 2015, doi: 10.1109/JSTQE.2014.2376697.

[7] B. Mayer et al., "Lasing from individual GaAs-AlGaAs core-shell nanowires up to room temperature," Nat. Commun., vol. 4, pp. 1-7, 2013, doi: 10.1038/ncomms3931.

[8] J. D. Joannopoulos, S. G. Johnson, J. N. Winn, and R. D. Meade, Photonic Crystals: Molding the Flow of Light, Second. Princeton University Press, 2008. 
[9] E. Yablonovitch, "Inhibited Spontaneous Emission in Solid-State Physics and Electronics," Phys. Rev. Lett., vol. 58, no. 20, pp. 2059-2062, May 1987, doi: 10.1103/PhysRevLett.58.2059.

[10] M. Notomi, E. Kuramochi, and H. Taniyama, "Ultrahigh-Q Nanocavity with 1D Photonic Gap,” Opt. Express, vol. 16, no. 15, p. 11095, Jul. 2008, doi: 10.1364/OE.16.011095.

[11] Y. Zhang et al., "Photonic crystal nanobeam lasers," Appl. Phys. Lett., vol. 97, no. 5, pp. 95-98, 2010, doi: 10.1063/1.3475397.

[12] K. Yao and Y. Shi, "High-Q width modulated photonic crystal stack mode-gap cavity and its application to refractive index sensing," Opt. Express, vol. 20, no. 24, p. 27039, Nov. 2012, doi: 10.1364/OE.20.027039.

[13] M. W. McCutcheon and M. Loncar, "Design of a silicon nitride photonic crystal nanocavity with a Quality factor of one million for coupling to a diamond nanocrystal," Opt. Express, vol. 16, no. 23, p. 19136, Nov. 2008, doi: 10.1364/OE.16.019136.

[14] A. Bazin et al., "Thermal management in hybrid InP/silicon photonic crystal nanobeam laser," Opt. Express, vol. 22, no. 9, p. 10570, May 2014, doi: 10.1364/OE.22.010570.

[15] K. Nozaki et al., "Femtofarad optoelectronic integration demonstrating energy-saving signal conversion and nonlinear functions," Nat. Photonics, vol. 13, no. 7, pp. 454-459, Jul. 2019, doi: 10.1038/s41566-019-0397-3.

[16] H. Altug, D. Englund, and J. Vučković, "Ultrafast photonic crystal nanocavity laser," Nat. Phys., vol. 2, no. 7, pp. 484-488, Jul. 2006, doi: 10.1038/nphys343.

[17] S. Matsuo et al., "High-speed ultracompact buried heterostructure photonic-crystal laser with 13 fJ of energy consumed per bit transmitted," Nat. Photonics, vol. 4, no. 9, pp. 648654, Sep. 2010, doi: 10.1038/nphoton.2010.177.

[18] H. Kim et al., "Monolithic InGaAs Nanowire Array Lasers on Silicon-on-Insulator Operating at Room Temperature," Nano Lett., vol. 17, no. 6, pp. 3465-3470, 2017, doi: 10.1021/acs.nanolett.7b00384. 
[19] S. T. Jagsch et al., "A quantum optical study of thresholdless lasing features in high- $\beta$ nitride nanobeam cavities," Nat. Commun., vol. 9, no. 1, p. 564, Dec. 2018, doi: 10.1038/s41467018-02999-2.

[20] K.-Y. Jeong et al., "Electrically driven nanobeam laser," Nat. Commun., vol. 4, no. 1, p. 2822, Dec. 2013, doi: 10.1038/ncomms3822.

[21] G. Bjork and Y. Yamamoto, "Analysis of semiconductor microcavity lasers using rate equations," IEEE J. Quantum Electron., vol. 27, no. 11, pp. 2386-2396, 1991, doi: $10.1109 / 3.100877$.

[22] Y. Yamamoto, S. Machida, and G. Björk, "Microcavity semiconductor laser with enhanced spontaneous emission," Phys. Rev. A, vol. 44, no. 1, pp. 657-668, Jul. 1991, doi: 10.1103/PhysRevA.44.657.

[23] M. Takiguchi et al., "All-Optical InAsP/InP Nanowire Switches Integrated in a Si Photonic Crystal," ACS Photonics, vol. 7, no. 4, pp. 1016-1021, Apr. 2020, doi: 10.1021/acsphotonics.9b01705.

[24] M. Takiguchi et al., "A hybrid nanowire photo-detector integrated in a silicon photonic crystal," in Conference on Lasers and Electro-Optics, 2019, vol. 1, no. 111, p. SM4J.3, doi: 10.1364/CLEO_SI.2019.SM4J.3.

[25] H. Schmid et al., "Template-assisted selective epitaxy of III-V nanoscale devices for coplanar heterogeneous integration with Si," Appl. Phys. Lett., vol. 106, no. 23, p. 233101, Jun. 2015, doi: 10.1063/1.4921962.

[26] M. Borg et al., "Vertical III-V nanowire device integration on Si(100)," Nano Lett., vol. 14, no. 4, pp. 1914-1920, 2014, doi: 10.1021/n1404743j.

[27] M. Borg, H. Schmid, K. E. Moselund, D. Cutaia, and H. Riel, "Mechanisms of templateassisted selective epitaxy of InAs nanowires on Si," J. Appl. Phys., vol. 117, no. 14, p. 144303, Apr. 2015, doi: 10.1063/1.4916984.

[28] D. Cutaia, K. E. Moselund, H. Schmid, M. Borg, A. Olziersky, and H. Riel, 
"Complementary III-V heterojunction lateral NW Tunnel FET technology on Si," in 2016 IEEE Symposium on VLSI Technology, Jun. 2016, pp. 1-2.

[29] S. Mauthe et al., "Ultra-Thin III-V Photodetectors Epitaxially Integrated on Si with Bandwidth Exceeding 25 GHz," in Optical Fiber Communication Conference (OFC) 2020, 2020, p. M3D.3, doi: 10.1364/OFC.2020.M3D.3.

[30] M. Borg et al., "High-Mobility GaSb Nanostructures Cointegrated with InAs on Si," ACS Nano, vol. 11, no. 3, pp. 2554-2560, 2017, doi: 10.1021/acsnano.6b04541.

[31] S. Adachi, "Optical dispersion relations for GaP, GaAs, GaSb, InP, InAs, InSb, Al x Ga 1x As, and In 1-x Ga x As y P 1-y," J. Appl. Phys., vol. 66, no. 12, pp. 6030-6040, Dec. 1989, doi: 10.1063/1.343580.

[32] B. R. Bennett, R. A. Soref, and J. A. Del Alamo, "Carrier-induced change in refractive index of InP, GaAs and InGaAsP," IEEE J. Quantum Electron., vol. 26, no. 1, pp. 113-122, 1990, doi: $10.1109 / 3.44924$.

[33] Z. Wang et al., "Room-temperature InP distributed feedback laser array directly grown on silicon," Nat. Photonics, vol. 9, no. 12, pp. 837-842, 2015, doi: https://doi.org/10.1038/nphoton.2015.199.

[34] S. Mauthe et al., "High-speed III-V nanowire photodetector monolithically integrated on Si," Nat. Commun., pp. 1-7, doi: 10.1038/s41467-020-18374-z. 